\title{
Microscopic Characteristics and Modelling of Pedestrian Inflow Process with Inactive Persons
}

\author{
Xinyu Fan · Long Xia · Weiguo Song \\ State Key Laboratory of Fire Science, University of Science and Technology of China, \\ Hefei, China, \\ E-mail: fan1025@mail.ustc.edu.cn, qwfzpk@mail.ustc.edu.cn,wgsong@ustc.edu.cn
}

Received: 4 October 2021 / Last revision received: 4 October 2021 / Accepted: 4 November 2021 DOI: $10.17815 / \mathrm{CD} .2021 .136$

\begin{abstract}
Inflow and outflow processes are common phenomena in daily life. Many types of research have been conducted to study the features of the outflow process, especially in scenarios with a single room or a straight corridor. A few scholars have paid attention to the movement characteristics of pedestrian inflow. Further explorations are still under great demand. In this contribution, a set of pre-conducted experiments are used to analyze the characteristics of the pedestrian inflow process with inactive persons. In these experiments, inactive persons were required to randomly cease within the room, leading to intensive detour behavior of pedestrians. The characteristics are carefully investigated using gradient analysis and curl analysis. To mimic the aforementioned inflow process, static global field is constructed to heuristically navigate a social force based microscopic model. The proposed model can reproduce the self-organized phenomena in the experiments. Our work can help understand the field feature of the pedestrian inflow process with inactive persons. High chaos level areas can be marked out providing practical information for managers.
\end{abstract}

Keywords Inflow process $\cdot$ pedestrian dynamics $\cdot$ modelling $\cdot$ simulation

\section{Introduction}

With a rapidly growing global population, excessively large crowds and congestion have become serious problems in both developed and developing countries. To mitigate this, numerous studies have been conducted to understand the behavior of pedestrian crowds 
and their evacuation processes [1-3]. A lot of studies focused on getting pedestrian movement characteristics, such as evacuation time [4-6], pre-movement time [7], stepping behaviors [8], density-flow relation [9-11].

Evacuation process presents the form of people escaping from the inside of a certain area to the outside, which can be called 'outflow'. The inflow process is defined by successive entry of pedestrians into a confined area and their subsequent dwelling [12]. However, there is no definite destination for people in the inflow process and they have to distribute themselves in the certain area. The study of the inflow process can be beneficial to the safety of people's lives because it occurs so frequently in our daily life under different scenarios [13].

Ezaki et al. [14]summarized several distinct phases of inflow process of a pedestrian: perception, decision making for destination, interaction between other pedestrians, stopping, direction change, and adaptation to incoming people. Moreover, they found four dominant factors: flow avoidance, distance cost, angle cost and boundary preference which affected the decision making in inflow process [15]. Liu et al. [16] conducted pedestrian inflow experiments in a square room and adopted several analytical methods, e.g. the Voronoi diagram method, proxemics, and point pattern analysis to investigated the spatial distribution of pedestrians at steady state. Qi et al. [17] studied the effect of the initial ratio of boarding to alighting group size on the average alighting time and proposed a cellular automata model to simulate passenger alighting and boarding behavior.

Until now, many mathematical models have been proved to be able to efficiently characterize pedestrian crowds during evacuation [18-20]. Specifically, the cellular automaton (CA) model [21-23] and the lattice-gas model [24-26] are proposed, which in general divide the evacuation room into proportionally proportioned cells containing individuals. However, the models restrains the movement direction of pedestrian flow [27]. To overcome this shortcoming, the social force model (SFM) characterized by continuous dynamics was proposed [28]. The SFM is based on the principle that pedestrian behavior is guided by 'social forces' which include both physical and psychological forces. The SFM also points out that pedestrian movement is induced not only by people's desire to reach a certain destination, but also by some other environmental factors. In order to describe more realistic scenarios, extended social force models have received increasing attention in recent years [29-31]. However, compared to the outflow process, the inflow process has been rarely investigated using modelling method. Therefore, it is worthwhile to be dedicated to uncovering the fundamental mechanism and underlying evolutionary dynamics of people's behavior in inflow process.

In this paper, we present a series of pedestrian experiments in a rectangular room to reveal the features of the inflow process, and the effect from different conditions of the experiments(with different ratio of inactive people), the characteristics of pedestrian spatial distribution and the congestion level are investigated. Then, we improved the original SFM to investigate the characteristics in inflow process.

The remainder of this paper is organized as follows. In Sec. 2 we describe the experimental setup. The quantitative results and analysis are given in Sec. 3, Sec. 4 and Sec. 5. In Sec. 6 we introduce the modelling methods. Finally, we conclude and discuss our results in Sec. 7. 


\section{Experimental setup}

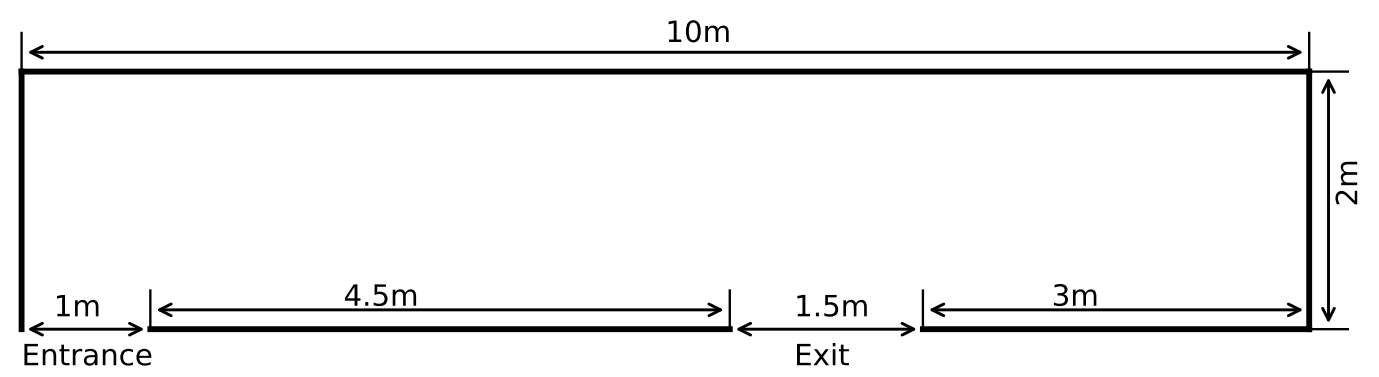

(a)
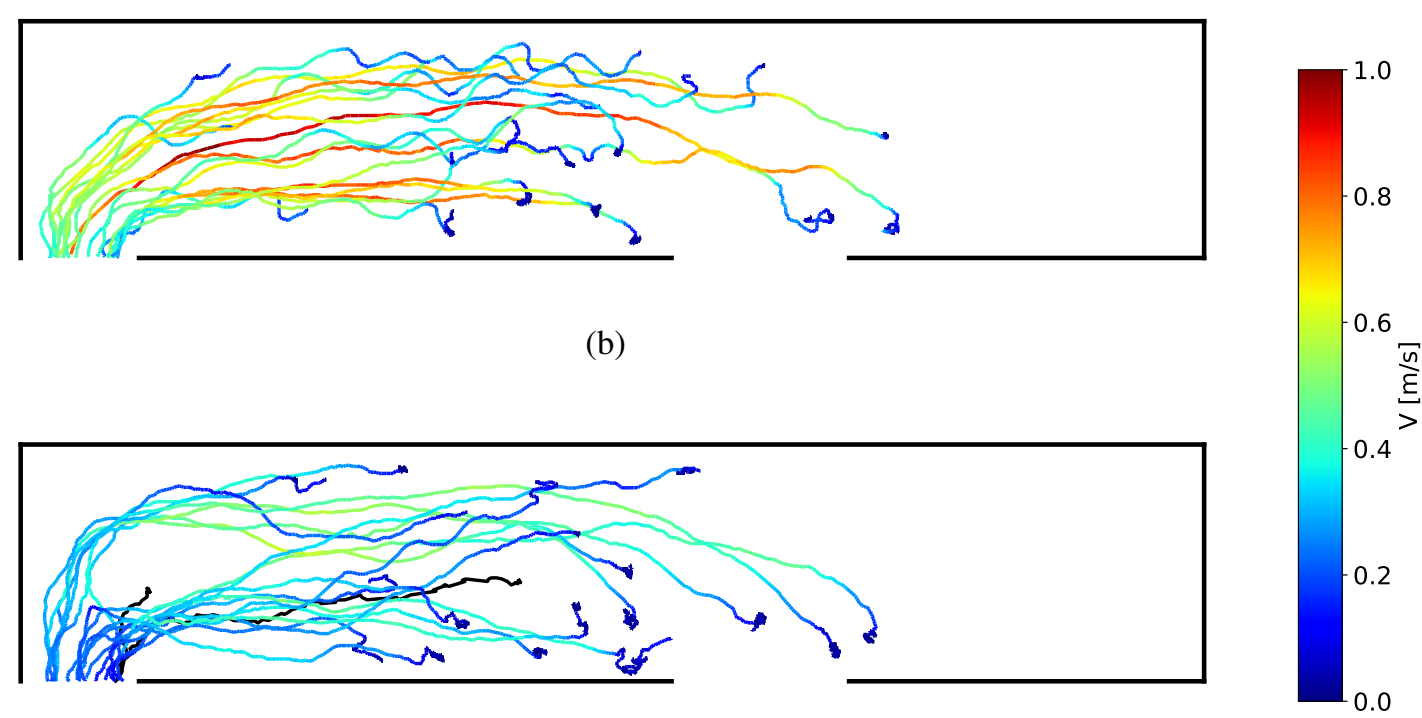

(b)

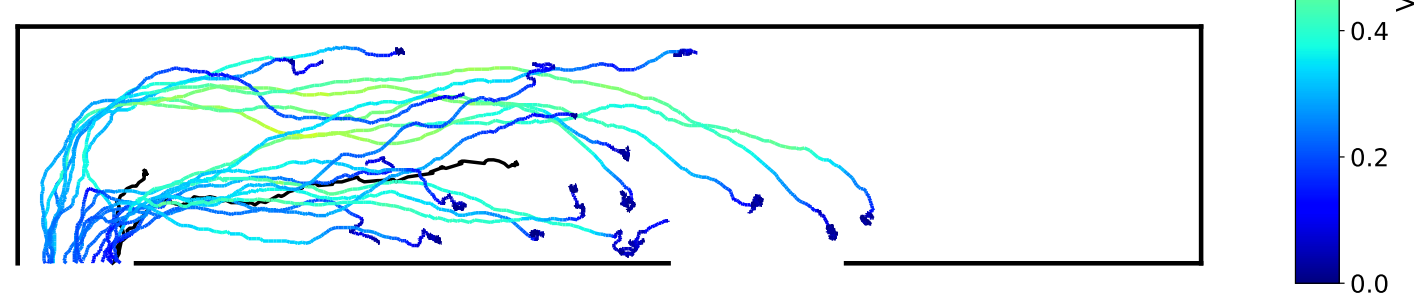

(c)

Figure 1 (a) The geometry of the experiment; (b) Trajectories of pedestrians in one trial without inactive persons; (c) Trajectories of pedestrians in one trial with 2 inactive persons marked with black.

The experiment was conducted in a rectangular room, whose length is $10 \mathrm{~m}$ and width is $2 \mathrm{~m}$. The entrance is in the front and the exit is near the middle. Particularly, the width of the entrance and the exit are $1 \mathrm{~m}$ and $1.5 \mathrm{~m}$ respectively, as shown in Fig. 1(a). 20 volunteers participated in the experiment, and the ratio of males to females was 1:1. The volunteers were informed to behave as normal in advance, temporarily without thinking of emergency situations. There were two types experiments:(1) without inactive persons and (2) with inactive persons. All the experiments included the same number of participants, but the number of inactive persons was changed in different runs(the number of inactive persons could be 2, 4, 6, 8, and 10).

In scenario 1, no inactive persons existed: participants waited outside the entrance and did not move until they were informed to enter the room. When they entered the room, 
they were allowed to stop at any position. Note that participants had the opportunities to change their positions due to their uncomfortable feelings while others entered the room. When all participants stopped moving, the inflow process finished.

However, inactive persons played different roles in scenario 2. In analog to scenario 1, the active participants gathered outside the room, then when the experiment began, they behaved as same in scenario 1. Some participants were informed to be inactive in advance, which means they stood in front of the others, and entered the room ahead of active persons. The inactive persons were not capable of moving once they had chosen the position to stop, preventing others from moving freely, respectively. Just like pedestrians in public transportation, due to their personal reasons, some of them have no desire to move again. Fig. 1(b) and Fig. 1(c) show snapshots of one experiment without inactive persons and one with inactive persons. Tab. 1 shows the experimental condition in each scene.

\begin{tabular}{ccc}
\hline Scene name & Ratio of inactive persons & Repetition \\
\hline Experiment 1 & $0 \%$ & 3 \\
Experiment 2 & $10 \%$ & 3 \\
Experiment 3 & $20 \%$ & 4 \\
Experiment 4 & $30 \%$ & 3 \\
Experiment 5 & $40 \%$ & 3 \\
Experiment 6 & $50 \%$ & 3 \\
\hline
\end{tabular}

Table 1 Experimental condition in each scene.

\section{Inflow features}

The goal of our experiments was to elucidate how inactive persons influence pedestrians and the flow characteristics. Consequently, to examine this observation closely, we focus on each pedestrian's characteristics parameters, such as steady time, distance to the entrance, relation between densities and velocities.

\subsection{Distance to the entrance and steady time}

Because the aim of the experiments was to allow pedestrians to cease at the locations they felt comfortable, so we plot everyone's distance to the entrance against time from start. Apparently, at the beginning, the distance increase with time, then remain invariant. Therefore, we regard it as two stages and a piece-wise linear function is utilized to estimate the trend of the raw data. The fitting results are shown in Fig. 2(b).

The steady time implies how long each pedestrian spent until he or she stayed still, as shown in Fig. 2(a). It can be observed that when the proportion of inactive persons increases, the steady time varies at the same trend. We consider it could be affected by the difference in the target positions and the disturbance from the ceased inactive people. On 
the one hand, before each pedestrian enters the room, he/she is likely to determine his/her target position. The existence of inactive persons tends to change others' choices, consequently having an effect on the inflow time, whose increase may directly contribute to the phenomenon of bypassing, turn-around and decelerating behavior, relatively costing more time.

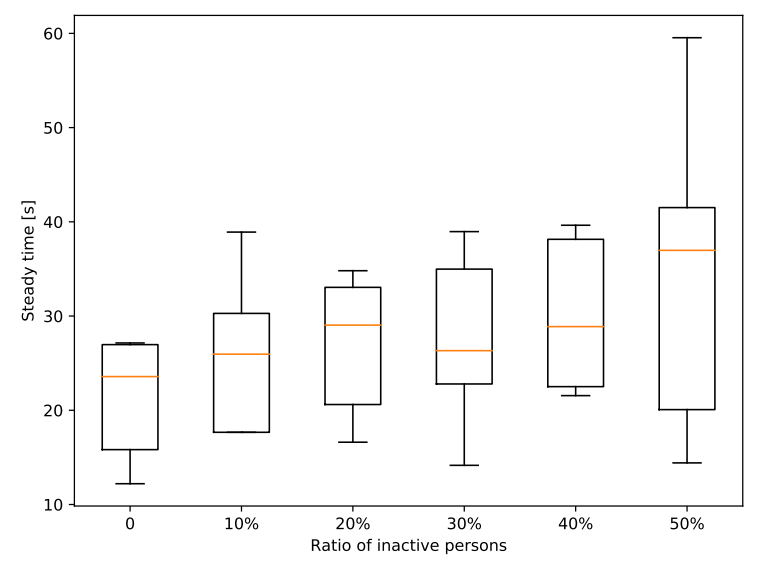

(a)

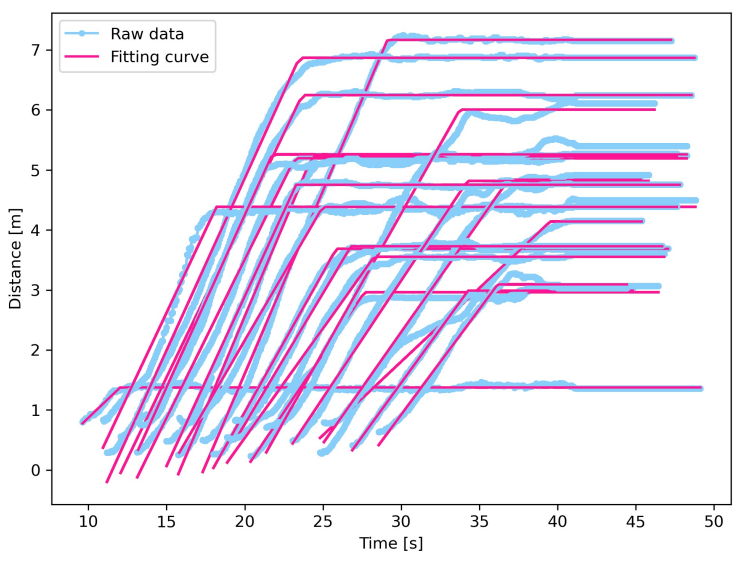

(b)

Figure 2 (a) The steady time of each experiment; (b) Time evolution of the distance to the room entrance with 2 inactive persons. While the blue circles represent the raw data, the pink curves represent the fitting data.

\subsection{Fundamental diagram}

We analyze the data among all pedestrians occupying the room from pedestrians' trajectory data. We measure the velocity of pedestrian $i$ at a given time $t$ through a derivative of i's trajectory $s_{i}(t)$ :

$$
v_{i}(t)=\frac{s_{i}(t+\Delta t / 2)-s_{i}(t-\Delta t / 2)}{\Delta t}
$$

$\Delta t$ is the time interval, and $s_{i}(t)$ is the location of pedestrian $\mathrm{i}$ at time $\mathrm{t}$, here $\Delta t$ is set as $1 \mathrm{~s}$.

We calculate the local density on the basis of Voronoi method [32]: at each moment, pedestrians equally shared the public space around them. According to this, every pedestrian' Voronoi can be acquired and contribute to the local density correspondingly. Furthermore, it can be calculated by the following equations:

$$
\rho(\vec{x})=\left\{\begin{array}{l}
\frac{1}{\mid A_{i}}, \vec{x} \text { in polygon } i \\
0, \text { others }
\end{array}\right.
$$

$A_{i}$ is the area of polygon $i$. An example of the Voronoi cells of corresponding pedestrians is shown in Fig. 3, in a trial with 10 inactive persons (the ratio of the inactive persons is 
$50 \%$. )

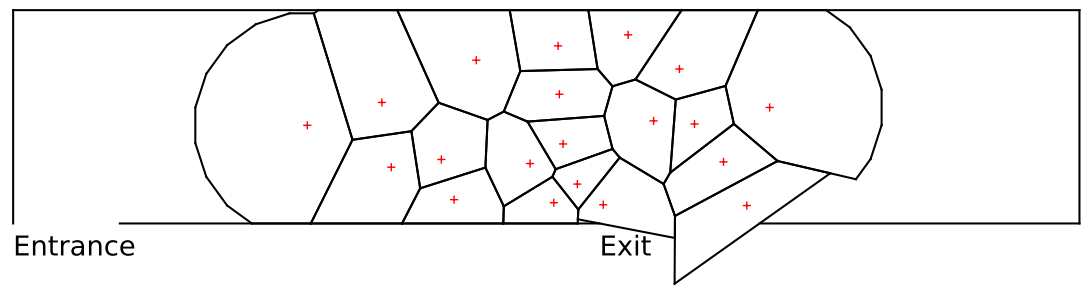

Figure 3 The Voronoi cells of corresponding pedestrians. While the black edges represent the area occupied by each pedestrian, the red marker inside the edges denote the position of the each pedestrian.

Fig. 14 shows the fundamental diagrams, wherein the pink lines represents the relationship between the average velocity and density. With the increase in density, the speed of pedestrians firstly decreases then becomes flat. We can observe that when there is no congested flow, the average speed of pedestrians with the most inactive people(50\% inactive persons) is clearly higher than that of others.

Nevertheless, in the same scenarios, compared with other cases, with the uptrend of the density, the downtrend of average velocity become stronger, as shown in Fig. 4(a). Also, in most range of the density, the velocity of ratio $30 \%$ seems to obtain a relative lower value. What we can imply from Fig. 4(b) is that with the ratio of inactive persons increasing, the average velocity varies randomly. A possible reason for this may be that the microscopic movement of experimental participants was not dramatically influenced by the role of the others (active or inactive). However, there seems to be no obvious correlation, so we conduct a correlation test. The average speed under different density is firstly normalized to a range from 0 to 1 . Therefore, the correlation between speed and the inactive ratio can be tested. The Pearson correlation coefficient between the ratio of inactive persons and average velocity is -0.231 , not significant at the 0.076 level ( bilateral). The result shows there is no significant correlation.

\section{The spatial distribution analysis}

As mentioned above, the distribution of pedestrians is clearly an import factor in determining the whole process. Since pedestrians remain steady when they finish their inflow process, we conduct distribution analysis to investigate which aspect affects pedestrians' distribution more significantly.

Additionally, the local density at place $\overrightarrow{\boldsymbol{r}}(x, y)$ at time $\mathrm{t}$ measured as [33]:

$$
f\left(\overrightarrow{\boldsymbol{r}}_{j}-\overrightarrow{\boldsymbol{r}}\right)=\frac{1}{\pi R^{2}} \exp \left[-\left\|\overrightarrow{\boldsymbol{r}}_{j}(t)-\overrightarrow{\boldsymbol{r}}\right\|^{2} / R^{2}\right]
$$




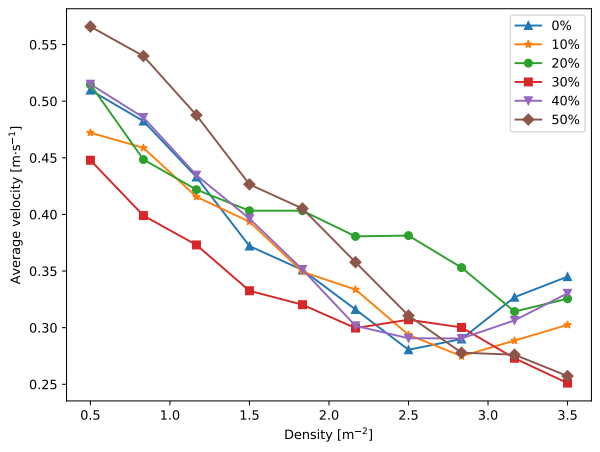

(a)

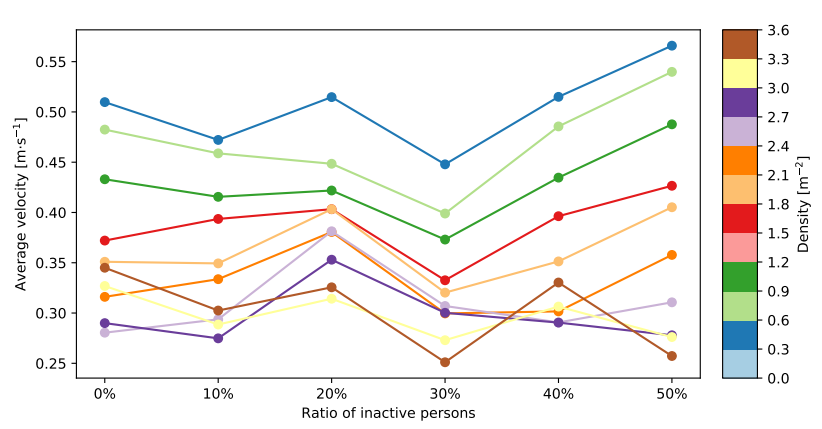

(b)

Figure 4 (a) The relationship between average velocity and density in different scenarios; (b) The average velocity in different scenarios among different density.

$$
\rho(\overrightarrow{\boldsymbol{r}}, t)=\sum_{j} f\left(\overrightarrow{\boldsymbol{r}}_{j}(t)-\overrightarrow{\boldsymbol{r}}\right)
$$

Here, $\overrightarrow{\boldsymbol{r}}_{j}(t)$ is the position of pedestrian $\mathrm{j}$ in the surrounding of $\overrightarrow{\boldsymbol{r}}$. $\mathrm{R}$ is a measurement parameter, and is set as 0.7 .

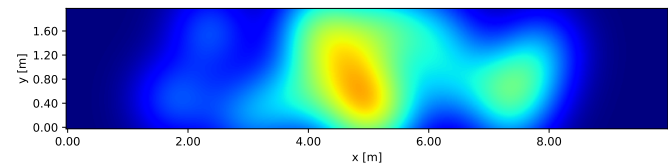

(a) $0 \%$ of inactive persons

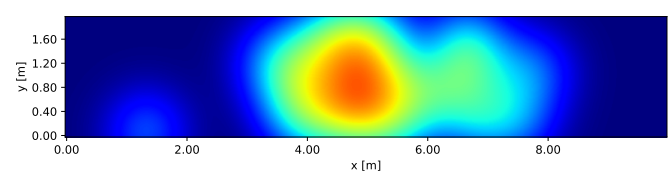

(c) $20 \%$ of inactive persons

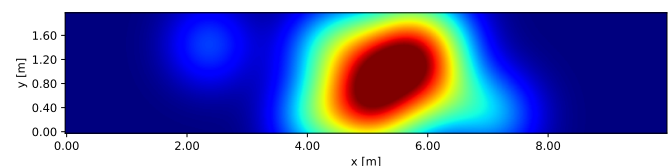

(e) $40 \%$ of inactive persons

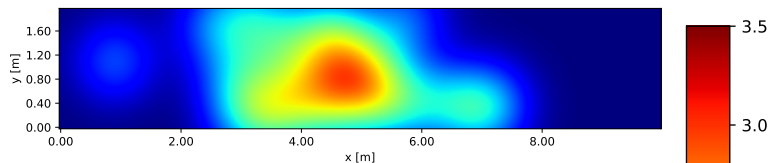

(b) $10 \%$ of inactive persons

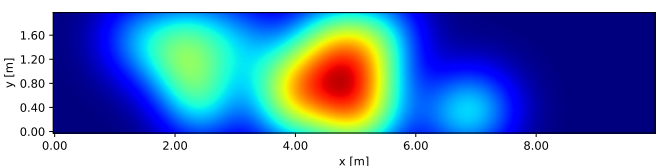

(d) $30 \%$ of inactive persons

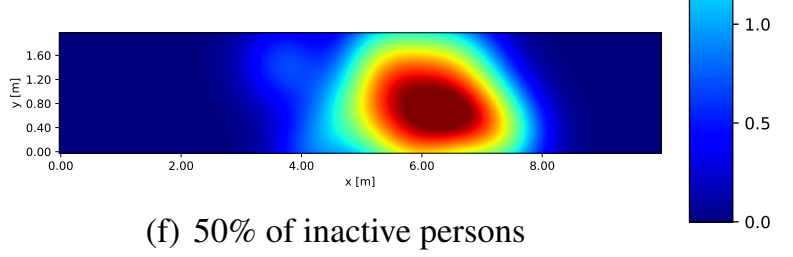

(f) $50 \%$ of inactive persons

Figure 5 The distribution of pedestrians in the steady state in different scenarios.

As noted, the density maps display different patterns for high and low ratios pf inactive persons (Fig. 5). For the high ratios, one can see a circular region of high density, while for the other, the surrounding regions have a relatively low density. What's more, we would like to stress that the density in the high ratios scenario reaches peak values higher than that of the others. This highlights that the distributions in high ratios show more approximately symmetry. 
When inactive people exist, people avoid the surrounding region, a feature reflected by notably lower values of the density in this region. On the contrary, with the ratio increasing, the densities around the inactive people in zones closer to the crowds are remarkably high. This implies that, whereas in a scenario with low ratio of inactive people, the most important magnitude defining the distribution is the distance to the exit. Instead, in high ratio scenarios, approaching the inactive crowds favors higher densities. This effect is observed independently on the ratio degree although, as expected.

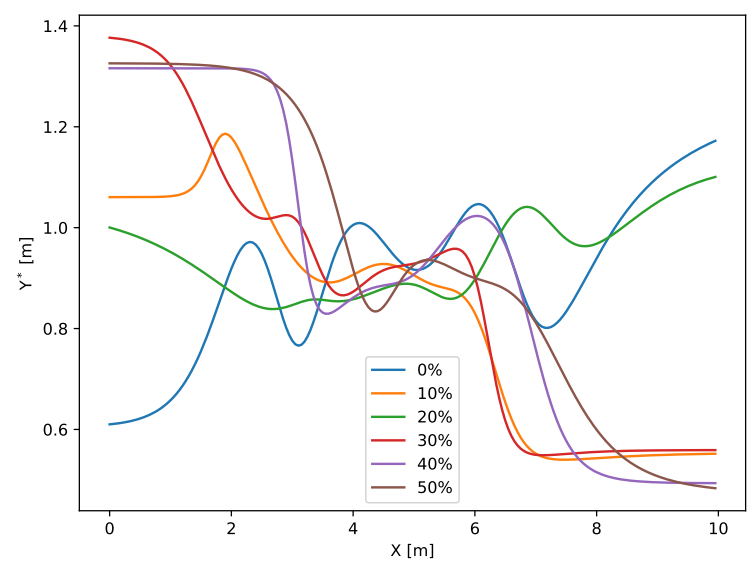

Figure 6 The distribution of pedestrians in the steady state in different scenarios along $\mathrm{x}$ axis.

Besides, using the local density as explanatory variables, we conduct an analysis about the variance of location along $\mathrm{x}$ axis, whose results is displayed in Fig. 5, where the vertical axis represents the $Y^{*} . Y^{*}$ is defined as:

$$
Y^{*}(x)=\frac{\int y \rho(x, y) d y}{\int \rho(x, y) d y}
$$

There is a distinct relation that the higher the ratio of inactive people are, the larger the varieties of $Y^{*}$ are. Therefore, it might be in agreement with the results of densities that is mentioned above. The uniform distributions are more likely to occur in a respectively low ratio than one that occur in a high ratio.

This suggests that pedestrians are more willing to move far away forming clusters, which is triggered by a block of inactive people, preventing faster pedestrians from walking at their desired speed. However, this effect is thought to be weaker when there is a larger group of inactive people.

\section{Congestion level}

To analyze the congestion of the pedestrians, we consider congestion level, which provides relatively an accurate analysis of macroscopic congestion [34,35]. To some extent, levels of congestion tend to imply how disordered and unorganized in crowds and are 
relevant to its rotation of the velocity vector field [36]. To better quantify the contribution of the local speed to the local curl, we define the congestion level as:

$$
\begin{gathered}
C l=\max \left(r_{z}^{*}\right)-\min \left(r_{z}^{*}\right) \\
\vec{R}(x, y)=\left(\begin{array}{c}
r_{x} \\
r_{y} \\
r_{z}
\end{array}\right)=\overrightarrow{\boldsymbol{\Delta}} \times \overrightarrow{\boldsymbol{v}}(x, y) \\
r_{z}^{*}=\frac{r_{z}}{|\overline{\boldsymbol{v}}|}
\end{gathered}
$$

Here, $|\bar{v}|$ is the average velocity calculated in the measurement area.

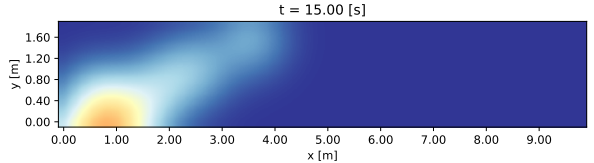

(a) $\mathrm{t}=15.00 \mathrm{~s}$

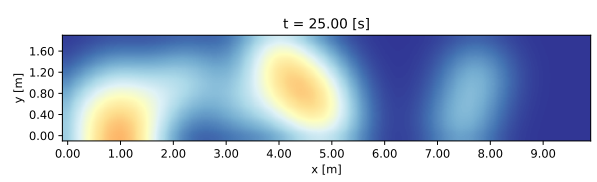

(c) $\mathrm{t}=25.00 \mathrm{~s}$

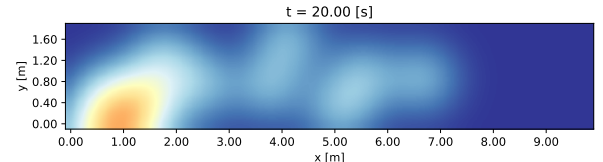

(b) $\mathrm{t}=20.00 \mathrm{~s}$

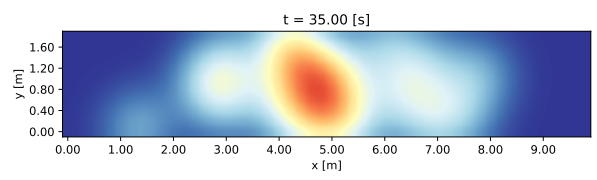

(d) $\mathrm{t}=35.00 \mathrm{~s}$

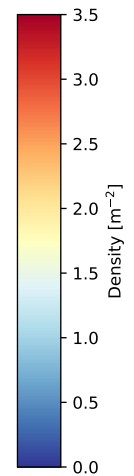

Figure 7 Density maps for experiment of $20 \%$ ratio of inactive persons explored.

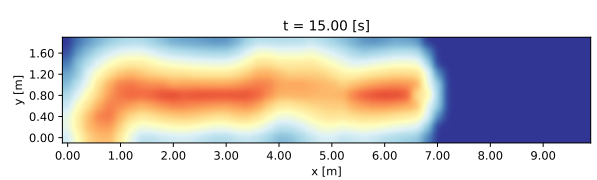

(a) $\mathrm{t}=15.00 \mathrm{~s}$

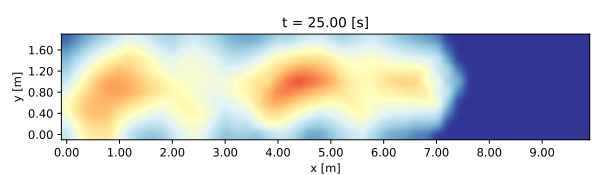

(c) $\mathrm{t}=25.00 \mathrm{~s}$

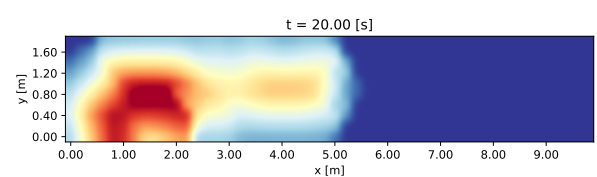

(b) $\mathrm{t}=20.00 \mathrm{~s}$

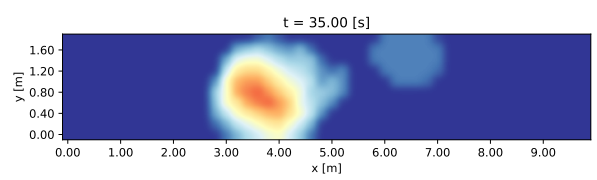

(d) $\mathrm{t}=35.00 \mathrm{~s}$

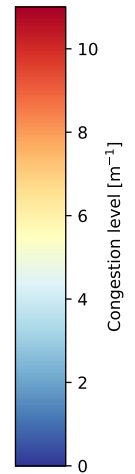

Figure 8 Congestion level maps for experiment of $20 \%$ ratio of inactive persons explored.

Over a wide range of time intervals, results of congestion level and density are computed. Fig. 7 and Fig. 8 display an example with 4 inactive persons in the experiment (The ratio of inactive persons is $20 \%$. ).

By comparing the density map of Fig. 7 with the congestion level map of Fig. 8, the higher density in Fig. 7 is observed near the exit of the experimental area where pedestrians are 
more likely to pass while moving inside the room. While the lower densities are observed around the entrance, which constitutes the location with the higher degree of congestion. Apparently, the higher density is found close to the exit by moving away from the entrance, and increasing values for congestion level are observed. The congestion level map generally seems to be translated a small distance from the entrance to the center of exit.

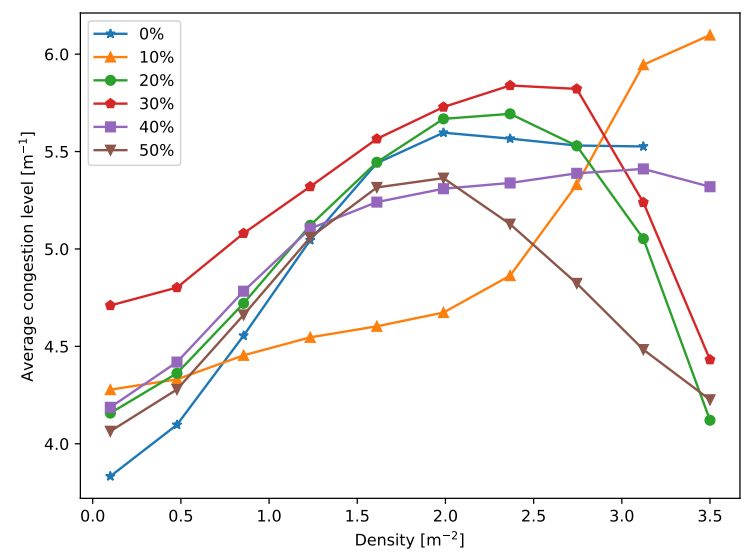

Figure 9 Relations between average congestion level and local density.

In addition, to perform a more detailed analysis on the relationship of different quantities with density, scatter plots have been created combining the density in each location with the corresponding congestion level. To be noted, the calculation of congestion level is based on the small time steps, therefore, we obtain the average results among a continuous range of density. As Fig. 9 clearly shows, the peak of congestion level is much higher in the low ratio cases. There is a tendency toward a decrease among most of cases, however, due to the limited data, we couldn't distinguish the exact density. The low ratio cases appear to be slightly less congested, although the difference only appears at lower density.

Finally, to study further and compare them with different scenarios, we analyze the time evolution of maximum congestion level for all experimental conditions. Results for this type of analysis are presented in Fig. 15, it is clearly seen that peak values are quickly reached in most of the cases and the fluctuation varies. In addition, it is interesting to notice that pedestrians in low ratio cases are likely to take shorter time to stay organized and final values are still moderate.

\section{Model}

Microscopic simulation models enable the researchers to replicate a wide range of physical and psychological issues that people experience during an emergency evacuation. This study aims to find the optimum pattern for pedestrian inflow process. Hence, to implement the proposed idea, a framework is developed enhanced with Social Force Model that 
consists of three parts. The social force model is described as below [3]:

$$
m_{i} \frac{d \overrightarrow{\boldsymbol{v}}_{i}}{d t}=m_{i} \frac{v_{i}^{0}(t) \cdot \overrightarrow{\boldsymbol{e}}_{i}^{0}-\overrightarrow{\boldsymbol{v}}_{i}(t)}{\tau_{i}}+\overrightarrow{\boldsymbol{f}}_{i j}+\overrightarrow{\boldsymbol{f}}_{i W}
$$

In the first phrase, inner force to the destination is calculated, where pedestrian $\mathrm{i}$ with mass $m_{i}$ drives at a certain desired speed $v_{i}^{0}$ in a certain direction $\overrightarrow{\boldsymbol{e}}_{i}^{0}$, and his/her actual velocity $\overrightarrow{\boldsymbol{v}}_{i}$ tends to reach the desired speed in each reaction time $\tau_{i}$.

The second phrase represents the interaction force drilled between pedestrian $i$ and pedestrian $\mathrm{j}$, which is calculated:

$$
\overrightarrow{\boldsymbol{f}}_{i j}=\left(A_{i} \cdot e^{r_{i j}-d_{i j} / B_{i}}+k g\left(r_{i j}-d_{i j}\right)\right) \overrightarrow{\boldsymbol{n}}_{i j}
$$

The third phrase represents the interaction force between the individual $i$ and the surrounding walls.

$$
\overrightarrow{\boldsymbol{f}}_{i W}=\left(A_{i} \cdot e^{r_{i}-d_{i W} / B_{i}}+k g\left(r_{i}-d_{i W}\right)\right) \overrightarrow{\boldsymbol{n}}_{i W}-\kappa g\left(r_{i}-d_{i W}\right)\left(\overrightarrow{\boldsymbol{v}}_{i} \cdot \overrightarrow{\boldsymbol{t}}_{i W}\right) \overrightarrow{\boldsymbol{t}}_{i W}
$$

\subsection{Static field}

Let us study pedestrians' choice of location. By introducing static field, as shown in Fig. 10, the value of the static field is expressed as:

$$
S(\vec{r})=\left(\frac{2}{1+e^{0.6\left(d_{E x i t}-2\right)}}+1\right) d_{\text {Exit }}-d_{W}(\overrightarrow{\boldsymbol{r}})-\left(\frac{1}{1+e^{0.6\left(d_{\text {Entrance }}-2\right)}}+1\right) d_{\text {Entrance }}
$$

Here, $d_{\text {Exit }}$ and $d_{\text {Entrance }}$ represent each pedestrian's location's distance to the exit and the entrance, respectively. $d_{W}(\overrightarrow{\boldsymbol{r}})$ refers to the shortest distance from a specific point $\overrightarrow{\boldsymbol{r}}$ to the boundaries, as shown in Fig. 11.

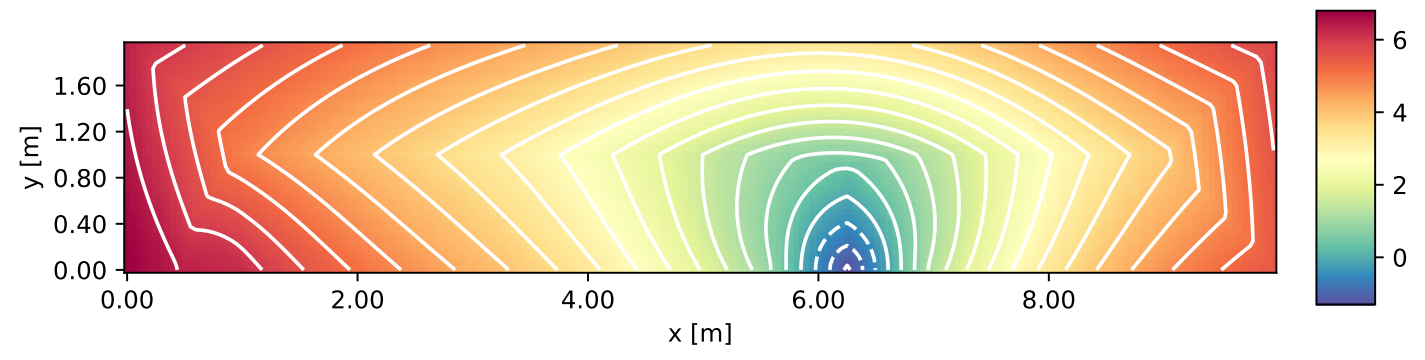

Figure 10 Static field concerning the influence of entrance and exit.

Firstly, based on the experiments, pedestrians gather outside the room and inactive people stand in front of the crowds. Then, when the simulation begins, the dominant factor pedestrians take into account differs. From active people's perspective, they alter their approach by choosing the surrounding location whose static value is lower, respectively. In terms of inactive people, on the one hand, they need to consider the static value. On the other hand, their terminations are combined with the experimental data. 


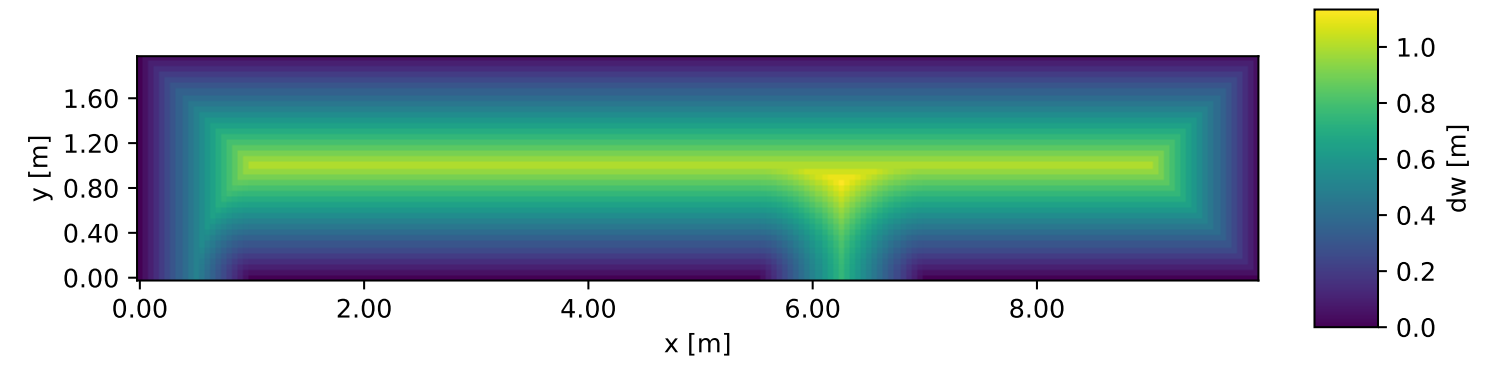

Figure 11 The value of $d_{W}$.

\subsection{Social force}

To implement the proposed idea, the locomotion model is designed, which consists of:

1. Inner force to destination;

2. Repulsive force among pedestrians;

3. Repulsive force and attractive force between pedestrians and walls;

4. Repulsive force and attractive force between pedestrians and exit.

Therefore, an individuals' locomotion is defined as below:

$$
m_{i} \frac{d \overrightarrow{\boldsymbol{v}}_{i}}{d t}=\overrightarrow{\boldsymbol{f}}_{s d}+\overrightarrow{\boldsymbol{f}}_{i j}+\overrightarrow{\boldsymbol{f}}_{i W}^{a}+\overrightarrow{\boldsymbol{f}}_{i W}^{r}+\overrightarrow{\boldsymbol{f}}_{i E}^{a}+\overrightarrow{\boldsymbol{f}}_{i E}^{r}
$$

The first part consists of traditional self-driven force in SFM, but, in contrary to traditional SFM, the desired direction is determined by static value. Moreover, inactive people receive an addition self-driven force, relevant to the final destination in experimental data:

$$
\overrightarrow{\boldsymbol{f}}_{s d}=m_{i} \frac{v_{i}^{0}(t) \cdot \overrightarrow{\boldsymbol{e}}_{i}^{s}-\overrightarrow{\boldsymbol{v}}_{i}(t)}{\tau_{i}}+2 m_{i} \frac{v_{i}^{0}(t) \cdot \overrightarrow{\boldsymbol{e}}_{i}^{d}-\overrightarrow{\boldsymbol{v}}_{i}(t)}{\tau_{i}}
$$

The third part and the fourth part are similar.

$$
\begin{aligned}
& \overrightarrow{\boldsymbol{f}}_{i W}=\left\{\begin{array}{l}
\overrightarrow{\boldsymbol{f}}_{i W}^{r}=\left(A_{i} \cdot e^{r_{i}-d_{i W} / B_{i}}+k g\left(r_{i}-d_{i W}\right)\right) \overrightarrow{\boldsymbol{n}}_{i W}-\kappa g\left(r_{i}-d_{i W}\right)\left(\overrightarrow{\boldsymbol{v}}_{i} \cdot \overrightarrow{\boldsymbol{t}}_{i W}\right) \overrightarrow{\boldsymbol{t}}_{i W} \\
\overrightarrow{\boldsymbol{f}}_{i W}^{a}=-30 / d_{i W} \overrightarrow{\boldsymbol{n}}_{i W}
\end{array}\right. \\
& \overrightarrow{\boldsymbol{f}}_{i E}=\left\{\begin{array}{l}
\overrightarrow{\boldsymbol{f}}_{i E}^{r}=\left(A_{i} \cdot e^{r_{i}-d_{i E} / B_{i}^{1}}+k g\left(r_{i}-d_{i E}\right)\right) \overrightarrow{\boldsymbol{n}}_{i E}-\kappa g\left(r_{i}-d_{i E}\right)\left(\overrightarrow{\boldsymbol{v}}_{i} \cdot \overrightarrow{\boldsymbol{t}}_{i E}\right) \overrightarrow{\boldsymbol{t}}_{i E} \\
\overrightarrow{\boldsymbol{f}}_{i E}^{a}=-60 / d_{i E} \overrightarrow{\boldsymbol{n}}_{i E}
\end{array}\right.
\end{aligned}
$$

Here, $\overrightarrow{\boldsymbol{f}}_{i E}^{r} / \overrightarrow{\boldsymbol{f}}_{i W}^{r}$ and $\overrightarrow{\boldsymbol{f}}_{i E}^{a} / \overrightarrow{\boldsymbol{f}}_{i W}^{a}$ represent the repulsive force and attractive force between pedestrians and exit/walls.

The numerical values of the constant parameters of the model are selected and presented in Tab. 2 


\begin{tabular}{cc}
\hline Parameter & Value \\
\hline Simulation parameters & \\
$v_{i}^{0}$ & $1 \mathrm{~m} / \mathrm{s}$ \\
$\tau_{i}$ & $0.5 \mathrm{sec}$ \\
$A_{i}$ & $2000 \mathrm{~N}$ \\
$B_{i}$ & $0.08 \mathrm{~m}$ \\
$B_{i}^{1}$ & $0.02 \mathrm{~m}$ \\
$\mathrm{k}$ & $12 \times 10^{5} \mathrm{~kg} / \mathrm{s}^{2}$ \\
$\kappa$ & $2.4 \times 10^{5} \mathrm{~kg} / \mathrm{m} \cdot \mathrm{s}$ \\
Pedestrian specification & \\
$m_{i}$ & {$[75-85] \mathrm{kg}$} \\
$r_{i}$ & $0.25 \mathrm{~m}$ \\
$\mathrm{~N}$ & 20 \\
\hline
\end{tabular}

Table 2 Parameters of the model.

\subsection{Simulation and results}

With the aim of confirming the validity of our model, we simulate three models in comparison:

1. SFM;

2. SFM + STATIC FIELD;

3. Our proposed model.

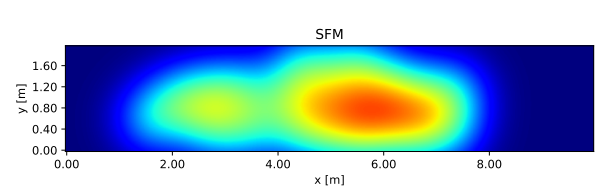

(a)

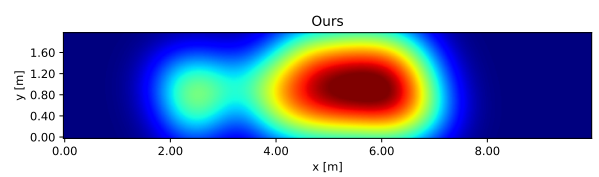

(c)

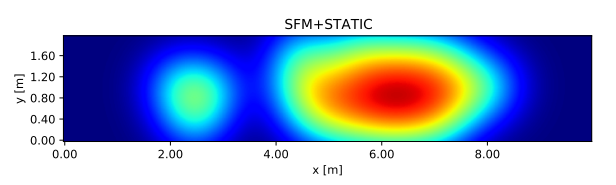

(b)

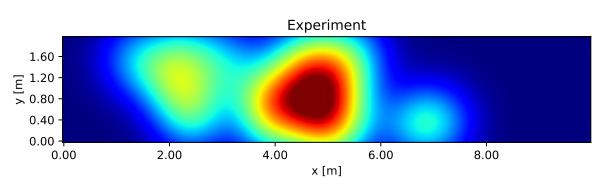

(d)

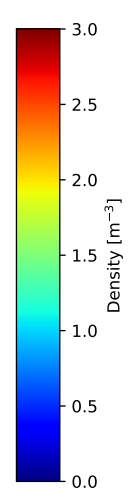

Figure 12 The distribution of pedestrians in the steady state with 8 inactive persons. (a) The results of SFM; (b) The results of SFM + STATIC; (c) The results of our proposed model; (d) The results of experiment.

An example of the distributions in steady time are shown in Fig. 12. Besides, we calculate the value of $Y^{*}(x)$ mentioned in Sec. 3, and investigate RMSE, as plotted in Fig. 13 where 


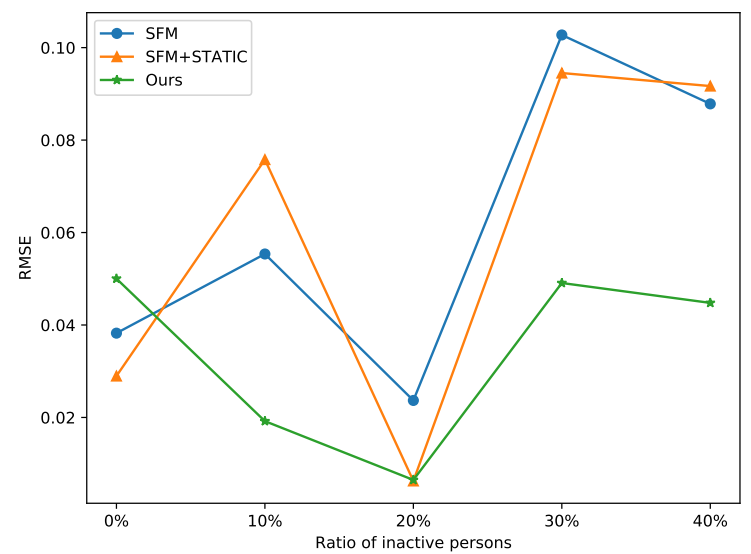

Figure 13 Simulation results for the proposed model in comparison to the experimental scenarios

\begin{tabular}{cccccc}
\hline Ratio of inactive persons & $0 \%$ & $10 \%$ & $20 \%$ & $30 \%$ & $40 \%$ \\
\hline SFM & 0.038 & 0.055 & 0.024 & 0.10 & 0.09 \\
SFM + STATIC FIELD & $\mathbf{0 . 0 2 9}$ & 0.076 & 0.063 & 0.095 & 0.092 \\
Our proposed model & 0.050 & $\mathbf{0 . 0 1 9}$ & $\mathbf{0 . 0 0 6}$ & $\mathbf{0 . 0 4 9}$ & $\mathbf{0 . 0 4 5}$ \\
\hline
\end{tabular}

Table 3 RMSE of $Y^{*}$ in comparison to the experimental scenarios.

the vertical axis represents the Root Mean Squared Error (RMSE) of $Y^{*}(x)$ in comparison with the experimental data and the horizontal axis represents the scenarios.

Additionally, the value of each RMSE is displayed in the following Tab. 3 and the bold words represent the minimum. It is inferred from this result that in most scenarios, our proposed model is a more optimal method.

Considering the fact that for most types of pedestrian streams, the inflow nature of interactions among pedestrians is of enormous significance, our attempts to add this ingredient into a model are helpful in reproducing inflow self-organization phenomena.

\section{Conclusion}

In this study, the properties of pedestrian inflow into a room (with one entrance and one exit) were investigated in a series of experiments with enough space. The experiments were divided into two conditions: without inactive persons and with the presence of inactive persons.

Several characteristics of the inflow process are revealed. When it comes to the steady time, quantities of factors contribute to its varieties, consisting of decision-making, movement, avoidance and direction change. It can be inferred from the experiments that pedestrians will more likely to cost more time until they remain steady when the number of inactive persons increase, thus causing changes in velocities. 


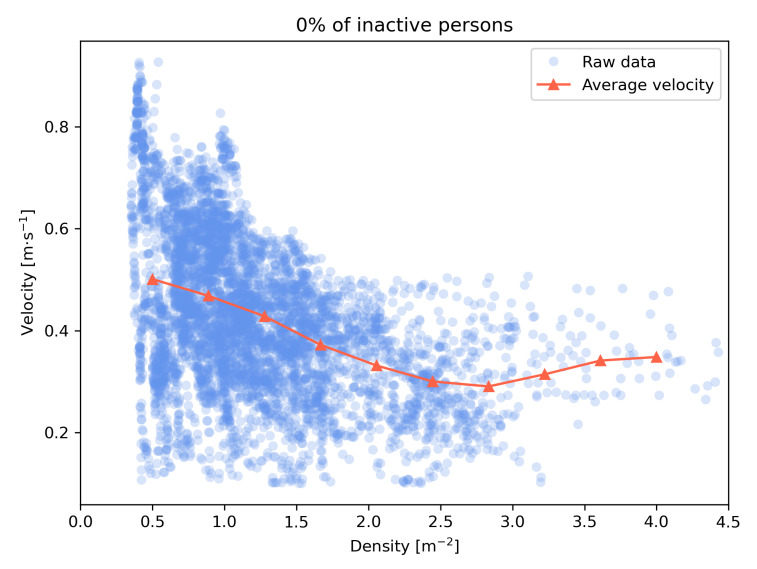

(a)

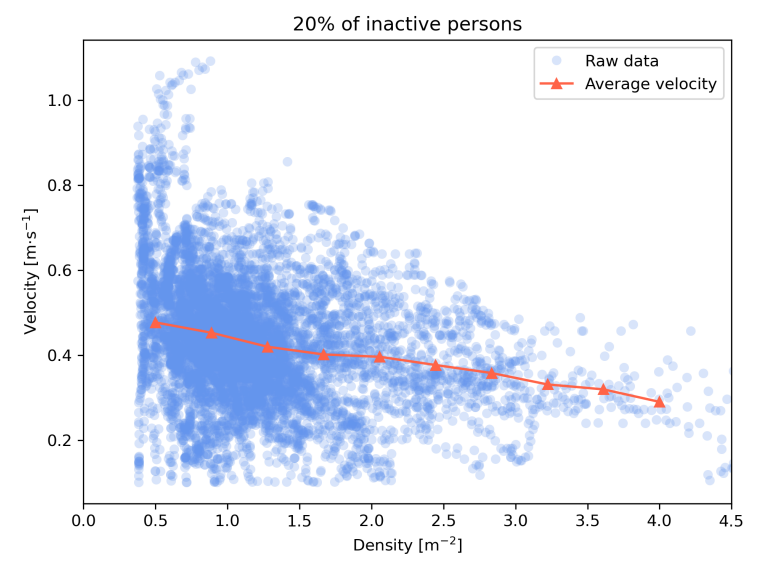

(c)

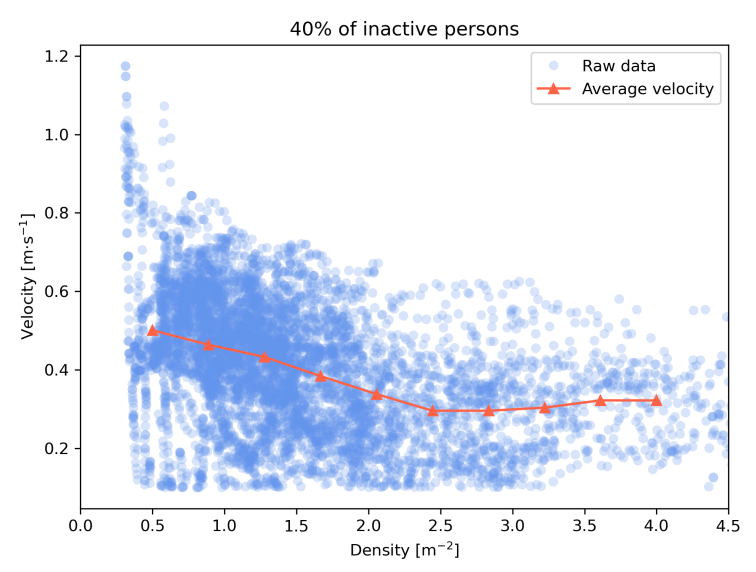

(e)

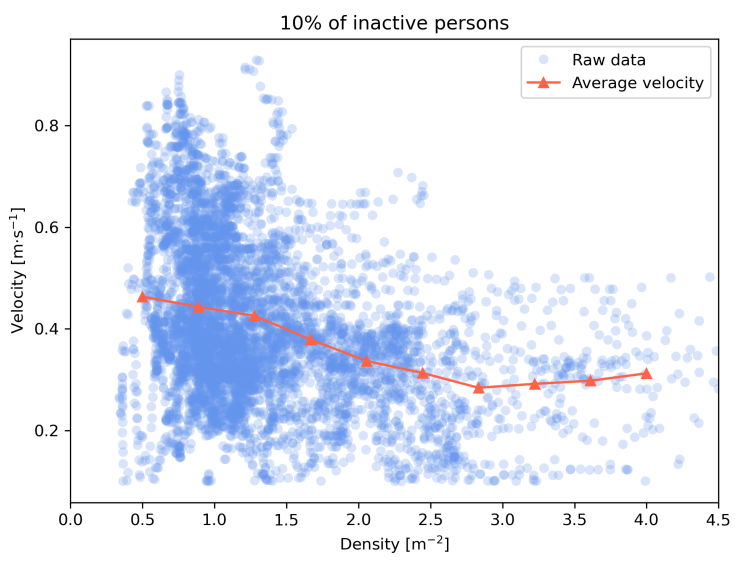

(b)

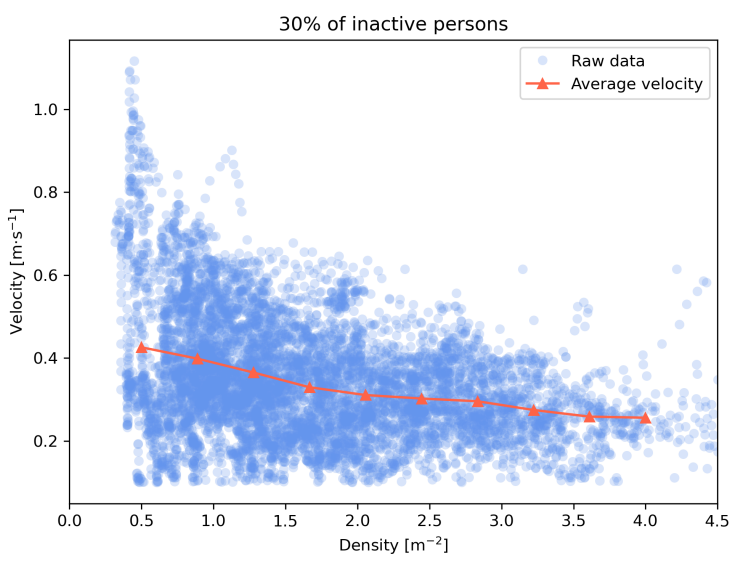

(d)

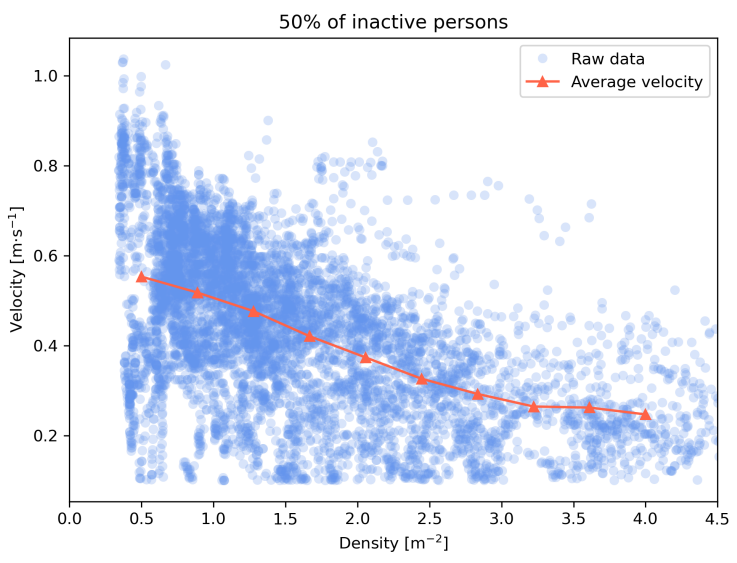

(f)

Figure 14 Fundamental diagrams in different experiments: (a) 0 inactive persons in the experiment; (b) 2 inactive persons in the experiment; (c) 4 inactive persons in the experiment; (d) 6 inactive persons in the experiment; (e) 8 inactive persons in the experiment; (f) 10 inactive persons in the experiment. 


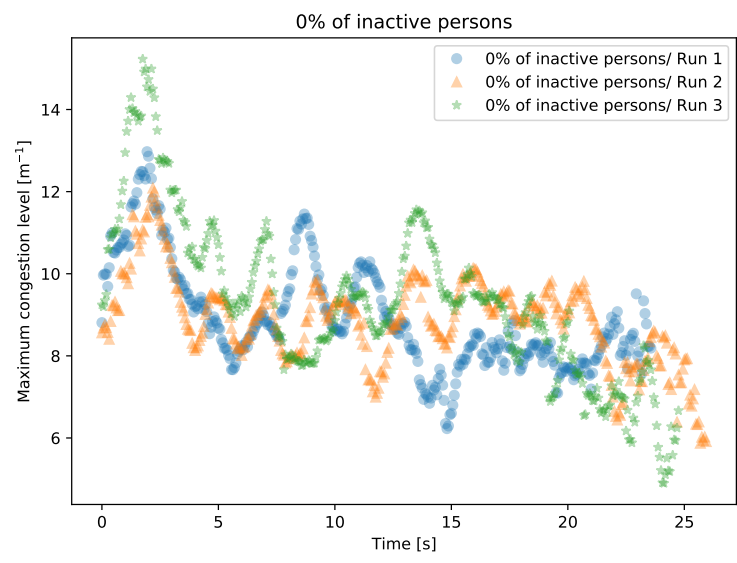

(a)

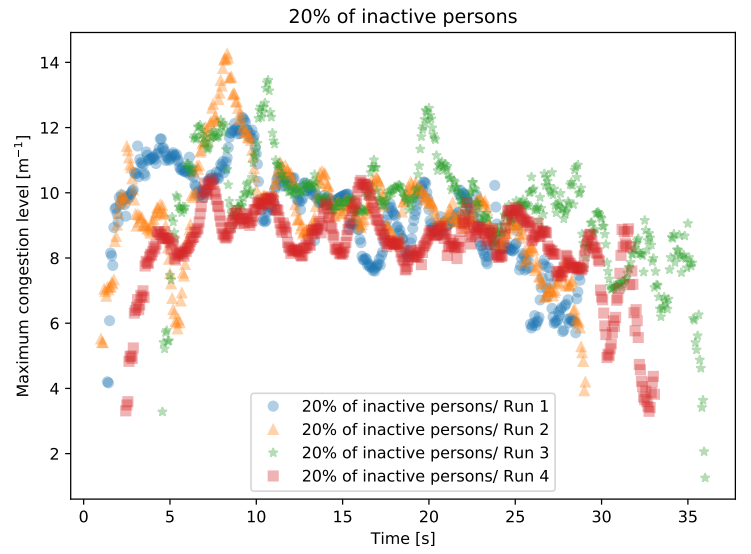

(c)

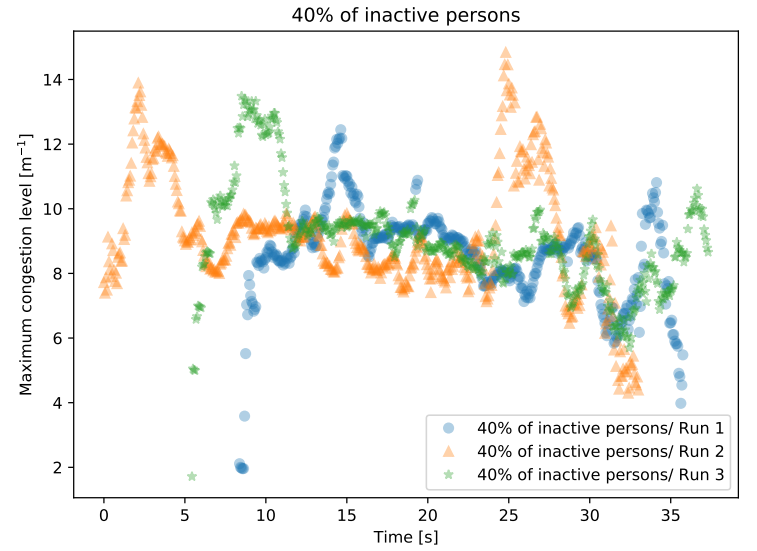

(e)

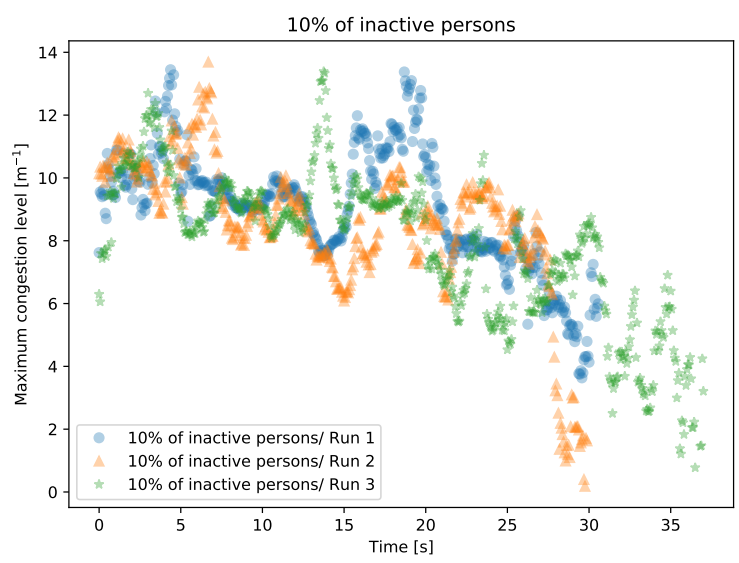

(b)

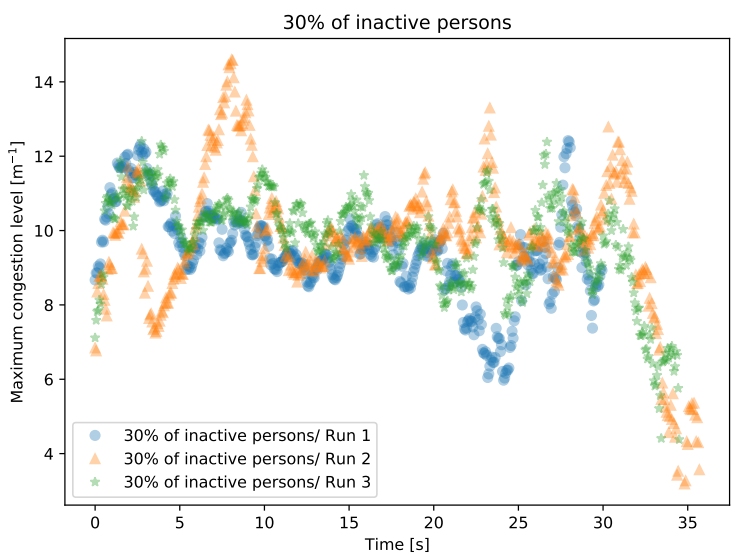

(d)

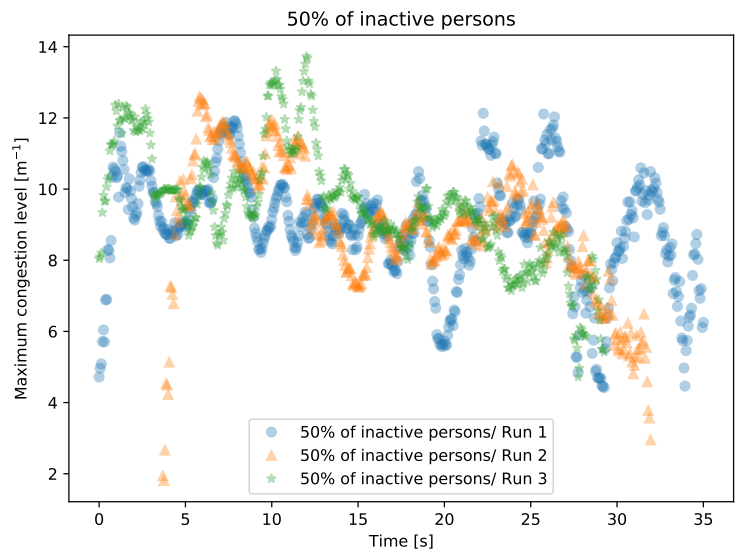

(f)

Figure 15 Time evolution of the maximum congestion level for different experiments. (a) 0 inactive persons in the experiment; (b) 2 inactive persons in the experiment; (c) 4 inactive persons in the experiment; (d) 6 inactive persons in the experiment; (e) 8 inactive persons in the experiment; (f) 10 inactive persons in the experiment. 
The study of the densities in the steady time has allowed us to consistently reveal the effect of inactive persons. The results suggest that pedestrians incline to gather around the center of the room where is near the entrance. Interestingly, with the increase of the inactive people, the densities of their surroundings increase consistently. The most possible reason can be concluded is that pedestrians receive more repulsion psychologically when standing near others, however, it becomes weaker in high ratio of inactive persona, respectively. Therefore, the inactive people determine more in pedestrian distribution, and in that sense the effect of inactive people should be taken into account.

As for the microscopic flow characteristics, the congestion level, to some extent, represent the severity of chaos. The higher density is found close to the exit by moving away from the entrance, and increasing values for congestion level are observed. Maybe, due to the limited data, while the peak of congestion level is much higher in the low ratio cases, there is a tendency toward a decrease among most of cases, and the low ratio cases appear to be slightly less congested, only appearing at lower density.

Finally, we have proposed a model based on social force model to simulate the inflow dynamics, which implements the interaction between exit and pedestrians. Also we focus on the static floor field, allowing pedestrians to choose their routes without much detour. Undoubtedly, the investigation of the model has achieved more optimal results in comparison with traditional social model.

It is very meaningful to study the characteristics of the pedestrian inflow since it occurs frequently in various kinds of facilities in our daily life. The obtained results give a contribution to a better comprehension of the dynamics of pedestrian inflow processes. As further research, we will make efforts to improve our model and conducting more experiments by changing experimental conditions. This task would be challenging, nevertheless, we believe it is a promising and worthwhile approach.

Acknowledgements This contribution is supported by National Natural Science Foundation of China (52074252), Anhui Key Research and Development Program(202004a07020052).

\section{References}

[1] Helbing, D., Mukerji, P.: Crowd disasters as systemic failures: analysis of the love parade disaster. EPJ Data Science 1(1), 1-40 (2012). do i : 10.1140 / ep jds 7

[2] Krausz, B., Bauckhage, C.: Loveparade 2010: Automatic video analysis of a crowd disaster. Computer Vision and Image Understanding 116(3), 307-319 (2012). doi:10.1016/j.cviu.2011.08.006

[3] Helbing, D., Farkas, I., Vicsek, T.: Simulating dynamical features of escape panic. Nature 407(6803), 487-490 (2000). doi: $10.1038 / 35035023$

[4] Fu, L., Song, W., Lo, S.: A fuzzy-theory-based behavioral model for studying pedestrian evacuation from a single-exit room. Physics Letters A 380(34), 2619-2627 (2016). doi:10.1016/j.physleta.2016.06.011 
[5] Zhang, J., Song, W., Xu, X.: Experiment and multi-grid modeling of evacuation from a classroom. Physica A: Statistical Mechanics and its Applications 387(23), 5901-5909 (2008). doi:10.1016/j.physa.2008.06.030

[6] Helbing, D., Buzna, L., Johansson, A., Werner, T.: Self-organized pedestrian crowd dynamics: Experiments, simulations, and design solutions. Transportation science 39(1), 1-24 (2005). doi:10.1287/trsc.1040.0108

[7] Guanquan, C., Jinhua, S.: The effect of pre-movement time and occupant density on evacuation time. Journal of fire sciences 24(3), 237-259 (2006). doi:10.1177/0734904106058249

[8] Kobes, M., Helsloot, I., de Vries, B., Post, J.: Exit choice,(pre-) movement time and (pre-) evacuation behaviour in hotel fire evacuation-behavioural analysis and validation of the use of serious gaming in experimental research. Procedia Engineering 3, 37-51 (2010). doi:10.1016/j.proeng.2010.07.006

[9] Seyfried, A., Steffen, B., Klingsch, W., Boltes, M.: The fundamental diagram of pedestrian movement revisited. Journal of Statistical Mechanics: Theory and Experiment 2005(10), P10002 (2005). doi:10.1088/1742-5468/2005/10/P10002

[10] Zhang, J., Klingsch, W., Schadschneider, A., Seyfried, A.: Ordering in bidirectional pedestrian flows and its influence on the fundamental diagram. Journal of Statistical Mechanics: Theory and Experiment 2012(02), P02002 (2012). doi:10.1088/1742-5468/2012/02/P02002

[11] Lian, L., Mai, X., Song, W., Richard, Y.K.K., Wei, X., Ma, J.: An experimental study on four-directional intersecting pedestrian flows. Journal of Statistical Mechanics: Theory and Experiment 2015(8), P08024 (2015). doi:10.1088/1742-5468/2015/08/P08024

[12] Ezaki, T., Yanagisawa, D., Ohtsuka, K., Nishinari, K.: Simulation of space acquisition process of pedestrians using proxemic floor field model. Physica A: Statistical Mechanics and its Applications 391(1-2), 291-299 (2012). doi:10.1016/j.physa.2011.07.056

[13] Fu, Libi, Fang, Zhiming, Liu, Xiaodong, Song, Weiguo: Experimental study of pedestrian inflow in a room with a separate entrance and exit. Physica A Statistical Mechanics and Its Applications (2016). doi:10.1016/j.physa.2015.09.026

[14] Ezaki, T., Ohtsuka, K., Yanagisawa, D., Nishinari, K.: Inflow process: A counterpart of evacuation. In: Traffic and Granular Flow'13, pp. 227-231. Springer (2015). doi:10.1007/978-3-319-10629-8_27 
[15] Ezaki, T., Ohtsuka, K., Chraibi, M., Boltes, M., Yanagisawa, D., Seyfried, A., Schadschneider, A., Nishinari, K.: Inflow process of pedestrians to a confined space. arXiv preprint arXiv:1609.07884 (2016). doi:10.17815/CD.2016.4

[16] Liu, X., Song, W., Fu, L., Lv, W., Fang, Z.: Typical features of pedestrian spatial distribution in the inflow process. Physics Letters A 380(17), 1526-1534 (2016). doi:10.1016/j.physleta.2016.02.028

[17] Zhang, Q., Han, B., Li, D.: Modeling and simulation of passenger alighting and boarding movement in beijing metro stations. Transportation Research Part C: Emerging Technologies 16(5), 635-649 (2008). doi:10.1016/j.trc.2007.12.001

[18] Tian, X., Li, K., Kang, Z., Peng, Y., Cui, H.: Simulating the dynamical features of evacuation governed by periodic vibrations. Chaos Solitons \& Fractals 139, 110099 (2020). doi:10.1016/j.chaos.2020.110099

[19] Min, H.E., Luan, Q.X., Shui, W.B., Hai-Ning, Y.U., Fan, D.: An improved social force model considering pedestrian perception avoidance feature of peer groups. Journal of Highway and Transportation Research and Development (2017)

[20] Wang, L., Zheng, J.H., Zhang, X.S., Zhang, J.L., Wang, Q.Z., Zhang, Q.: Pedestrians' behavior in emergency evacuation: Modeling and simulation. Chinese Physics B 25(11), 118901 (2016). doi:10.1088/1674-1056/25/11/118901

[21] Fu, Z., Jia, Q., Chen, J., Ma, J., Han, K., Luo, L.: A fine discrete field cellular automaton for pedestrian dynamics integrating pedestrian heterogeneity, anisotropy, and time-dependent characteristics. Transportation Research Part C Emerging Technologies 91(JUN.), 37-61 (2018). doi:10.1016/j.trc.2018.03.022

[22] Wang, J., Zhang, L., Shi, Q., Yang, P., Hu, X.: Modeling and simulating for congestion pedestrian evacuation with panic. Physica A: Statistical Mechanics and its Applications 428, 396-409 (2015). doi: $10.1016 / j$.physa.2015.01.057

[23] Liang, Chen, Tie-Qiao, Tang, Hai-Jun, Huang, Jian-Jun, Wu, Ziqi, Song: Modeling pedestrian flow accounting for collision avoidance during evacuation. Simulation modelling practice and theory: International journal of the Federation of European Simulation Societies 82, 1-11 (2018). doi:10.1016/j.simpat.2017.12.011

[24] Guo, X., Chen, J., Zheng, Y., Wei, J.: A mobile lattice gas model for simulating pedestrian evacuation. Physica A: Statistical Mechanics and its Applications 391(3), 582-592 (2012). doi:10.1016/j.physa.2007.10.001

[25] Wei, Y.F., Xue, Y., Dai, S.Q.: Small-Grid Analysis of Evacuation Processes with a Lattice Gas Model for Mixed Pedestrian Dynamics. Pedestrian and Evacuation Dynamics 2008 (2010). doi: $10.1007 / 978-3-642-04504-2 \_60$ 
[26] Zhang, Yi-Ming, Huang, Hai-Jun, Shang, Hua-Yan: An extended mobile lattice gas model allowing pedestrian step size variable. Physica, A. Statistical mechanics and its applications (2015). doi:10.1016/j.physa.2015.01.006

[27] Li, Q., Gao, Y., Chen, L., Kang, Z.: Emergency evacuation with incomplete information in the presence of obstacles. Physica A: Statistical Mechanics and its Applications 533 (2019). doi:10.1016/j.physa.2019.122068

[28] Helbing, D., Molnar, P.: Social force model for pedestrian dynamics. Phys.rev.e 51(5), 4282 (1995). doi:10.1103/PhysRevE.51.4282

[29] Liu, Qian: The effect of dedicated exit on the evacuation of heterogeneous pedestrians. Physica A: Statistical Mechanics and its Applications 506, 305-323 (2018). doi:10.1016/j.physa.2018.04.032

[30] Han, Y., Liu, H.: Modified social force model based on information transmission toward crowd evacuation simulation. Physica A Statistical Mechanics \& Its Applications 469, 499-509 (2016). doi: 10.1016/j.physa.2016.11.014

[31] Echeverría-Huarte, I., Zuriguel, I., Hidalgo, R.C.: Pedestrian evacuation simulation in the presence of an obstacle using self-propelled spherocylinders. PHYSICAL REVIEW E 102(1) (2020). doi:10.1103/PhysRevE.102.012907

[32] Steffen, B., Seyfried, A.: Methods for measuring pedestrian density, flow, speed and direction with minimal scatter. Physica A: Statistical mechanics and its applications 389(9), 1902-1910 (2010). doi:10.1016/j.physa.2009.12.015

[33] Helbing, D., Johansson, A., Al-Abideen, H.Z.: Dynamics of crowd disasters: An empirical study. Physical review E 75(4), 046109 (2007). doi:10.1103/PhysRevE.75.046109

[34] Feliciani, C., Murakami, H., Nishinari, K.: A universal function for capacity of bidirectional pedestrian streams: Filling the gaps in the literature. PloS one 13(12), e0208496 (2018). doi:10.1371/ journal.pone.0208496

[35] Feliciani, C., Nishinari, K.: Measurement of congestion and intrinsic risk in pedestrian crowds. Transportation research part C: emerging technologies 91, 124-155 (2018). doi:10.1016/j.trc.2018.03.027

[36] Fujita, A., Feliciani, C., Yanagisawa, D., Nishinari, K.: Traffic flow in a crowd of pedestrians walking at different speeds. Physical Review E 99(6), 062307 (2019). doi:10.1103/PhysRevE.99.062307 